\title{
Resultate einiger ophthalmometrischer und mikrooptometrischer Messungen.
}

Von

Dr. M. Reich aus Petersburg.

1872 ist in einer interessanten Arbeit von Mandelstamm und Schöler*) eine neue Methode zur Bestimmung der optischen Constanten des Auges veröffentlicht. Mit Hilfe dieser Methode, deren Grundzüge den Verfassern von Professor Helmholtz vorgeschlagen wurden, wird die Entfernung zwischen dem Hornhautscheitel und der vorderen Linsenfläche (Pupillarebene) einerseits und zwisehen ersterem und dem Scheitel der hinteren Linsenfläche anderseits - zwei Grössen, deren Kenntniss zur Berechnung der optischen Constanten und Cardinalpunkte des Auges ebenso wichtig ist, als eine absolut genaue Bestimmung derselben schwierig war - mit Hilfe eines Mikroskops genauer, viel einfacher und rascher ausgeführt, als mit Hilfe der früheren, ebenso geistreichen, aber complicirteren Methoden von $\mathrm{Helm}$ holtz**). Diese nene Methode stellt in der Ophthalmo-

*) Archir f. Ophthalm. Ba. XVIII. 2.

**) Arch. f. Ophth., Bd. I, 2; pag, 31.51 und and. Physiologische Optik. S. $18 \mathrm{u}$, and. 
motrie einen Fortschritt dax, in Folge dessen die Anwendung der letzteren auch für klinische Zwecke in manchen Hinsichten sehr wesentlich erleichtert wird. Ieh erlaube mir, das Wesentliche der Bestimmungsart beider obengenannten Grössen in wenigen Worten wiederzugeben.

Bestimmung des Abstandes der Pupillarebene vom Hornhautscheitel.

Ein modificirtes Mikroskop von Liebreich (Corneamikroskop) $)^{*}$ ) wird vor dem $z u$ untersuchenden, so viel wie möglich unbeweglichen Auge so eingestellt, dass der in das Mikroskop schauende Beobachter thunlichst scharf den Pupillarrand der Iris sieht. Darauf lässt man mit Hilfe einer sehr nahe vor dem Objectivende des Mikroskopes angeschraubten, um die verticale Axe drehbaren planparallelen Glasplatte, zwischen Cornea und letztere das Luftibild einer weit entiernten Gaslampenflamme fallen.

Dieses Bild, welches nur selten hinter die Glasplatte zu stehen kommt, wird durch eine Sammellinse (deren Brennweite am besten ungefahr $3 \frac{1}{2} \mathrm{zoll}$ ist) entworfen, welche zwischen der weit zur Seite stehenden Lampen. flamme und der Glaspiatte, der letzteren ziemlich nahe, aufgestellt wird. Indem man nun die Linse hin und her bewegt, gelingt es leicht, das Luftbild der Flamme in eine solche Entfernung vor der Hornhaut zu bringen, dass das Spiegelbild des Luftbildes dem Beobachter sehr klar und scharf in der Pupillarebene erscheint, und also mit dem Irisrande zugleich deutlich gesehen wird. Darauf bestimmt man, nach Eutfernung des beobachteten Auges, in welcher Entfernung vom Objecte (oder irgend einem beliebigen Punkte) des Mikroskopes sich die scheinbare Pupillarebene und das Luftbild der Flamme

*) Bei Paetz und Flohr in Berlin kostet das ,Mikrooptometer" [ron Schoeler und Mandelstamm so genannt] 36 Thlr. 
befanden; die Differenz (da das Luftbild sehr selten hinter die Glasplatte kommt) beider Entfernungen drückt die Entfernung zwischen dem Luftbild und der scheinbaren Pupillarebene aus. Wenn z. B. das Luftbild (a) $15 \mathrm{Mm}$. von irgend einem Punkte des Objectives, die Pupillarebene (b) aber in einer Entfernung von $42 \mathrm{Mm}$. von demselben steht, so ist die Entfernung $(d=a b)$ zwischen Luftbild und Pupillarebene $=27 \mathrm{Mm}$. Wenn die Krümmung der Hornhaut bekannt, so findet man auch leicht die Entferung $x b$ zwischen der scheinbaren Pupillarebene (oder, was ungefähr dasselbe ist, der vorderen Linsenfläche) und dem Scheitel der Hornhaut. Es ist uns nämlich d bekannt, d. h. die Summe der Entfernungen des leuchtenden Gegenstandes (a) und seines Spiegelbildes in $b$ von der spiegelnden Fläche (der Hornhaut in $x$ ), deren Lage wir eben suchen. Wir können sagen $d=f_{1}+f_{2}$. Dann wird in der allgemeinen Formel der conjugirten Brenn weiten für spiegelnde Flächen

$$
\left.-\frac{1}{\mathrm{r}}=\frac{1}{\mathrm{f}_{1}}-\frac{1}{\mathrm{f}_{2}} *\right)
$$

für unseren Fall uns $r$ (der Krümmungshalbmesser) bekannt, und $f_{1}=d-f_{2}$ sein.

Wir erhalten nun die Formel $-\frac{2}{\mathrm{r}}=\frac{1}{\mathrm{~d}-\mathrm{f}_{2}}+\frac{1}{\mathrm{f}_{2}}$, aus welcher für $f_{2}(=x b)$ die nicht ganz bequeme Formel $f_{2}=-\frac{d}{2} \pm \frac{d}{2} \sqrt{1+\frac{r^{2}}{d^{2}}}$ erhalten wird. Letztere wird durch Annäherung, mit Hilfe des binomischen Lehrsatzes, in die zur Rechnung geeignetere Formel verwandelt:

$$
\mathrm{f}_{2}=\frac{\mathrm{r}}{2}\left(1-\frac{\mathrm{r}}{2 \mathrm{~d}}\right) \text {. }
$$

$\left\langle\mathrm{f}_{2}=\right.$ scheinb. Abstand der Pupillarebene vom Hornhautscheitel). 
Der wirkliche Abstand wird nun nach der bekannten Formel $\xi=\frac{\mathrm{xf}_{2} *}{\mathrm{x}+\mathrm{f}_{1}}$ ) berechnet.

Auf dem ophthalmologischen Congress in London (im August 1872) demonstrirte Donders ein Instrument (ein Fernrohr, dessen Bewegung sehr genau abgemessen werden kann', welches gleichfalls für die Bestimmung der Tiefe der Augenkammer bestimmt ist. Das Instrument wird auf die vordere Hornhautfläche (ein Calomelkörnchen auf derselben) eingestellt; sodanu ist die Bewegung, welche das Instrument anzeigt, beim Einstellen von der Hornhautoberfläche auf den Irisrand, die scheinbare Entfernung der vorderen Hornbautfäche von der vorderen Linsenfäche. Die Tiefe der Augenkammer fand sich auf diese Weise gemessen, merklich kleiner, als man bisher angenommen hatte. Um die Hornhautkrümmung zu messen, ist ausserdem noch ein kleiner Planspiegel erforderlich, welcher das Licht einer Flamme in das Auge des beobachteten Individuums reflectirt. Entfernt man das Licht hinreichend weit, so darf man anvehmen, dass das splegelbild desselben, das von der Hornhaut entworfen wird, sich nahezu in dem Brennpunkt ihrer Krümmungsform befindet. Misst man auf die angegebene Weise die Entfernung der vorderen Hornhautfiäche von diesem Hornhautspiegelbild, so findet man die Länge der Brennweite, welche mit 2 multiplicirt den Krümmungshalbmesser der Hornhaut giebt**).

Aus dieser Beschreibung sieht man, dass die neue Methode nach Helmholt $z$ die Tiefe der vorderen Kammer zu messen, sich wesentlich von der Donders'schen unterscheidet.

*) Helmboltz. Arch. f. Ophth. Bd. I. 2, pag. 35.

**) Klinische Monatsblätter $f$. Augenheilk. 1872, S. 300. 
Bestimmungdes Ortes der hin teren Linsenfläche.

In ziemlicher Entfernung von dem untersuchten Auge, welches einen bestimmten Punkt, ungefähr in der Richtung der Axe des Mikroscopes fixirt, wird eine helle Gaslampenflamme oder das Bild der Sonne so angebracht, dass ein deutlicher Reflex (auch verdoppelt durch ein nach oben oder unten brechendes Prisma vor dem Auge) von der hinteren Linsenfläche im Centrum der Pupille erscheint. Bei unveränderter Stellung des untersuchten Auges wird nun das Objectiv des Mikroskopes so gestellt, dass der Beobachter ein scharfes Reflexbild von der Hornhaut sieht; darauf wird bestimmt, um wie viel das Objectiv aus- oder eingeschraubt werden muss, um die Reflexbilder der hinteren Linsentläche (bei Ruhe und bei Accommodation) einzustellen. Auf diese Weise ist es nicht schwierig, auch den Abstand des HornhautreHexbildes von dem Reflexbilde der hinteren linsenfläche in beiden Fällen zu bestimmen. Wenn z. B. bei Einstellung des Spiegelbildes der Fornhaut das Objectiv auf 10,75 Millim. ausgeschraubt ist und, um das Reflexbild der hinteren linsenflache scharf einzustellen, man dasselbe ungefähr noch bis 11,5 Millim. hinausschrauben muss, so steht das Spiegelbild der Hornhaut 0,6 $\mathrm{Mm}$. vor dem Reflexbild der hinteren Linsentläche, denn $\left.75,0-74,4^{*}\right)=0,6$. Wenn im gegebenen Fall der Radius der Hornhautkrümmung gleich 7,4544 $\mathrm{Mm}$. ist, so steht das Hornhautspiegelbild der Flamme $3,7272 \mathrm{Mm}$. und das der binteren Linsenflache 3,7272 $+0,6=4,3272 \mathrm{Mm}$. hinter der Hornhautoberfläche.

Die mit Hilfe des Mikroskopes bestimmte Grösse (x) des Abstandes des Reflexes der hinteren Linsenflächt von der Hornhautoberfläche benutzen wir für die Be-

*) Bei den angeführten zwei Ausschraubungen des Objectives wird ein gespanntes Coconfadenkreuz in den Entfernungen 74,4 und $75,0 \mathrm{Mm}$. schart gesehen. 
rechnung des Abstandes (d) der hinteren Linsenfläche vom zweiten Hauptpunkte des Auges. Durch Rechnung (l. cit. pag. 175) finden wir die Formel

$$
d=\frac{(x+q-p) F_{2}}{x+q-p+F_{1}}
$$

in der $F_{1}$ und $F_{2}$ die Hauptbrennweiten des Auges, $p$ den Abstand des ersten Hauptpunktes ron der Hornhaut, $q$ die Brennweite des spiegelnd brechenden Systems der hinteren Linsenfläche bedeutet.

Der wirkliche Abstand der hinteren Linsenfläche von der Oberfläche der Hornhaut ist $=\mathbf{d}+\mathrm{p}+\varepsilon$, wo noch $\varepsilon$ den Abstand der beiden Hauptpunkte von einander bedeutet.

Das gefundene d wird zur Berechnung des Krüm. mungsradius der hinteren Linsenfläche, in die Formel $r=\frac{q\left(f_{2}-d\right)^{2}}{1 / 2 f_{1} f_{2}-q\left(f_{2}-d\right)}$ eingeführt.

Bei meinen Studien im Laboratorium des Herrn Professor Helmholtz habe ich einige detaillirte Messungen, sowohl mit dem Ophthalmometer, als anch nach der von Schoeler und Mandelstamm beschriebenen Methode ausgeführt, und erlaube mir die Resultate dieser Messungen zu veröffentlichen, da bis jetzt, so viel ich weiss, solche mit dem Mikroskop nur an zwei Augen bekannt geworden sind, und da ich auch die Gelegenheit benutzt habe, bei den Rechnungen die neuen, von Helmholtz neuerdings etwas modificirten Zahlen für die optischen Constanten und Cardinalpunkte des schematischen Auges anzuwenden. Ausserdem waren die von Schoeler und Mandelstamm zum ersten Male deutlich bewiesenen Ortsveränderungen des hinteren

*) Helmboltz Arch.f Ophth. Bd. I, 2; Knapp ibid. Bd. VI, 2. 
Linsenscheitels*) in zwei Augen bei der Accommodation so wichtig, dass eine Bestimmung derselben auch in anderen Augen wenigstens sehr interessant erschien. Endlich interessirten mich anch vergleichende Messungen mit dem Ophthalmometer und dem Mikroskop.

Ich führe die optischen Constanten und Cardinalpunktedes schematischen (accominodations losen) Auges nach Helmboltz an:

Brechungsvermögen der wässrigen

Mm.

und gläsernen Feuchtigkeit . $=1,3365$

Brechungsvermögen (totales) der

Krystallinse . . . . . . . . $=1,4371\left(\right.$ statt $\left.\frac{16}{11}\right)$

Krümmungsradius der Hornhaut $.=7,829$ (statt 8)

Desgl. der vorderen Linsenfläche $.=10,0$

Desgl. der hinteren Linsenfäche $.=6,0$

Ort der vorderen Linsenfläche . . $=3,6$

Ort der hinteren Linsenfläche . $=7,2$

Vordere Brennweite der Hornhant . $=23,266$

Hintere desgl. . . . . . . . . $=31,095$

Brennweite der Linse . . . . . $=50,671$ (statt 43,707)

Abstand des vorderen Hauptpunktes

der Linse von der vord. Fläche $=2,12$

Abstand des hinteren Hauptpunktes

der Linse von der hint. Fläche $=1,274$

Abstand der beiden Hauptpunkte

der Linse von einander . . . $=0,206$

Hintere Brennweite des Auges . $=20,719$

Vordere Brennweite des Auges . . = 15,5025

Ort des vorderen Brennpunktes. . $=13,7525$

Ort des ersten Hauptpunktes . $.=1,750$

*) Bei Thieren ist ein Zurü ckweichen der hinteren Linsenfläche bei der Accommodation schon 1868 von Hensen und Völckers (Experimente über den Mechanismus der Accommodation. Kiel) bewiesen. 
$\mathrm{Mm}$.

Ort des zweiten Hauptpunktes . . $=2,115$

Ort des ersten Knotenpunktes . . $=6,966$

Ort des zweiten Knotenpunktes . . $=7,331$

Ort des hinteren Brennpunktes $=22,834$.

Bei der Berechnung der optischen Constanten und Cardinalpunkte der von mir untersuchten Augen benutzte ich die Zahlen, welche bei Berechnung dieser Grössen für das accommodirte schematische (angeführte) Auge erhalten werden, wenn dabei für die Krümmungsradien der Linsenoberflächen 6 und 5,5 Millimeter beibehalten werden.

Die Bestimmung des Krümmungsradius der Hornhaut

führte ich aussschliesslich mit dem Ophthalmometer aus. Obgleich wohl diese Bestimmnng leicht und rasch auch mit Hilfe des Milrroskopes (sowohl nach der Donders'schen, als auch nach der von Schoeler und Mandelstamm beschriebenen Methode) gemacht werden kann, so werden die durch diese mikrooptometrische Methode erhaltenen Zahlengrössen doch immer, wenn auch nur sehr unbedeutend, weniger genau sein, als die mit Hilfe des Ophthalmometers gefundenen, denn die Unabhängigkeit der Messungen von, wenn auch nur geringen, Schwankungen*) des Auges ist ein absolut unersetzlicher Vorzug des Ophthalmometers. Ausserdem wird die Mannigfaltigkeit der Anwendung des letzteren wohl nicht von dem Mikroskop erreicht werden können.

Der Krümmungsradius der Hornhaut (im horizontalen Meridian) wurde gefunden:**)

*) Zwar sind die Spiegelbilder der Hornhaut, um den Krüm. mungsradius zu ermitteln, nur in der Schoeler und Mandelstamm'schen Methode zu messen. Die Bestimmung des Radius nach Donders geschieht, wie wir gesehen, ohne die Messung der Spiegelbilder zu brauchen.

**) Als Mittelwerth aus mehreren Messungen. 
bei Herrn H. (25 Jahre $\mathrm{M}^{1} / 20$ ) $=$ 7,201 Millimeter.

bei Herrn G. $\left(26\right.$ " $\left.\mathbf{M}^{1} / 60\right)=7,4544 \quad$ "

bei Herrn S. $(26, \quad M 1 / 19)=7,7939$ "

Die Bestimmung des Abstandes des Horn-

hautscheitels von der Pupillarebene

(Tiefe der vorderen Kammer) wurde sowohl mit dem Ophthalmometer (an 2 Augen bei Accommodation für die Ferne) als auch mit dem Mikroskop (an allen 3 Augen bei Accommodation für die Ferne und Nähe) ausgeführt:

Tiefe der vorderen Kammer.

Bei Ruhe

Bei Accommod.

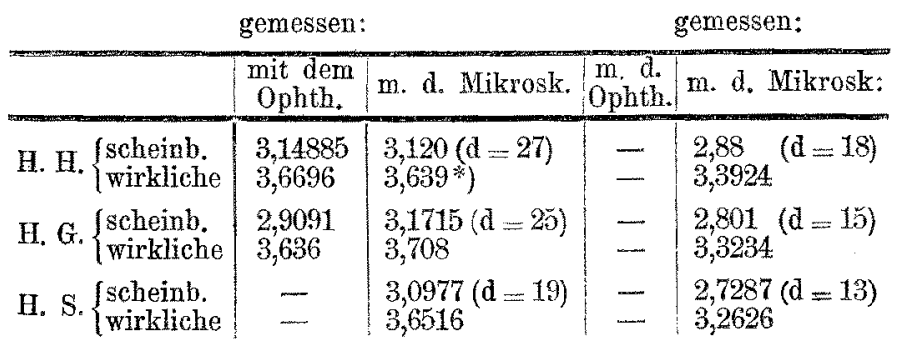

Bei den nachfolgenden Berechnungen ist die Tiefe der Kammer (Abstand der Pupillarebene von der Hornhautoberfläche) bei H. G. $=3,708$ bei Ruhe und $=3,3234$ bei Accommodation angenommen; denn bei mehreren Controlbestimmungen mit dem Mikroskop wurden nur ganz minimale Schwankungen erhalten, unmittelbar aufeinanderfolgende Bestimmungen mit dem Ophthalmometer ergaben relativ grosse Differenzen.

Der Grad der Ungenanigkeit der Bestimmung der Tiefe der Augenkammer vermittelst des Ophthalmo meters und die bedingenden Ursachen dieser Ungenauigkeit sind schon von Knapp**) und Schoeler**;

*) Bei der folgenden Berechnung ist, als Mittel 3,654 ange* nommen.

**) Ueber die Lage und Krümmung der Oberfläche der mensch. lichen Krystallinse etc. Arch. f. Ophth. Bd. VI. 2.

***ix) L. cit。 S. 159 . 
busprochen. Die sehr genaue Bestimmung der Tiefe der Kammer vermittelst des Mikroskopes ist schon daraus zu ersehen, dass bei Controlbestimmungen nur sehr geringe Abweichungen vorkommen. Die Schärfe oder Undeutlichkeit des Spiegelbildes in der Pupillarebene ist leicht zu controliren. Wenn z. B. gefunden wird $d=27$, bei einem Krümmungsradius der Hornhaut $=8,148$, so ist die scheinbare Tiefe $=3,4593$. Wenn bei Controlbestimmungen d wenig grösser oder wenig kleiner gefunden wird, so wird dadurch die Grösse für die Tiefe verhältnissmässig wenig beeinflusst. Wenn $d=27$, ist die scheinbare Tiefe $=3,4593$; bei $d=26$ ist $\mathrm{T} .=3,4356$; bei $\mathrm{a}=28$ ist $\mathrm{T} .=3,4812 ;$ bei $\mathrm{d}=29$ ist $\mathrm{T}=3,5016$. Die Schwankungen von $\mathrm{d}$ müssen auch gewöhnlich, wenn sie vorkommen, nicht mehr als 1 oder 2 Millimeter ausmachen. Je kleiner aber $d$ ist (z. B. bei Accommodation des Auges), einen desto grösseren Einfluss haben natürlich dieselben Schwankungsgrössen auf die berechnete Grösse der Augenkammertiefe.

Die Messungen der Krümmung der vorderen Linsenfläche wurden grösstentheils vermittelst des Ophthalmometers ausgeführt (mit Anwendung von Sonnenlicht) ${ }^{*}$ ), da jeder der nicht ganz scharf begrenzten Reflexe von dieser Fläche bei der starken Vergrösserung durch das Mikroskop im Sehfelde eine so grosse Aus. dehnung hatte, dass eine sehr genaue Bestimmung ( $\mathrm{Ab}$ messung) der Entfernung derselben mit dem Mikrometer, besonders im nicht accommodirten Auge, mir unmöglich war. Dazu gesellten sich noch einige Schwankungen des Auges, welche die Abmessung auch nur erschweren Konaten. Eine grössere Entfernung der Reflexe von ein-

*) Von Rosow (Petersburg) zuerst, dann von Woinow, Adamük, Schoeler angewendet. 
ander (eine Vergrösserung des gespiegelten Objectes also) mit der Absicht, den Abmessungsfehler zu verringern, würde aber das Resultat der Berechnung des Krümmungshalbmessers der Linsenfläche merklich beeinflussen.

Zur Messung der Spiegelbilder der vorderen Linsenfläche ziehe ich überhaupt das Ophthalmometer entschieden vor; die Sonnenreflexe von derselben waren nur in einem Auge etwas schwächer und verwaschen, waren aber überhaupt völlig lichtstark genug, auch bei Verdoppelung derselben durch die Ophthalmometerplatten.

Von der vorderen Linsenfläche wurden, nach der Angabe von Helmholtz zwei Reflexe entworfen: der cine von einem directen Sonnenbilde (im Focus einer Convexlinse, welche die vom Spiegel desHeliostaten reflectirten Sonnenstrahlen sammelte), der andere vermittelst eines Prismas, welches in einiger Entfernung vom Auge mit der brechenden Kante nach unten oder oben aufgestellt wurde. Aus der Grösse des Bildes ( $\beta$ ), der des Gegenstandes, (des Abstandes b zwischen den beiden Sonnenbildern) und der Entfernung $a$ des letzteren vom Auge wurde auf die übliche Weise die Brennweite $q$ des katoptrisch dioptrischen Systems vor der vorderen Linsenfläche berechnet. Nach Berechnung von $q$ wurde der Krümmungsradius der vorderen Linsenfläche nach der äblichen Formel

$$
\left.r=-\frac{q\left(f_{2}-d\right)^{2}}{1 / 2} f_{1} f_{2}+q\left(f_{2}-d\right)\right)
$$

berechnet. Bei der Messung der Spiegelbilder im accommodirten Auge wurde das Fixationszeichen entsprechend genähert.

*) Knapp. 1. cit. pag 33; Helmholtz. Archiv für Ophth. Bd. I, 2 , pag. 49 . 
Tabelle (II) zur Bestimmung des Krämmungsradiusdervorderen

Linsenfläche.*)
H. H.
H. $G$.
H. S.

Ophth. Mikr. Ophth. Mikr. Ophth. Mikr.

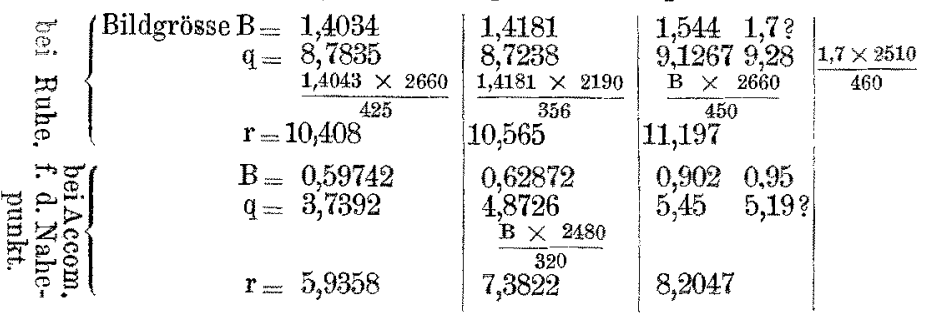

Bei den weiteren Berechnungen wurden nur die mit dem Ophthalmometer gewonnenen Zahlen für $\mathrm{B}$ benutzt, da eine genaue Messung mit dem Mikrometer, wie oben angegeben, mir ziemlich schwierig war. (S. die letzte Rubrik der Tabelle.)

Die zur Berechnung des Krümmungshalbmessers der hinteren Linsenfläche nöthigen Messungen wurden sowohl mit dem Ophthalmometer als auch mit dem Mikroskop ausgefübrt, wobei Sonnenreflexe benutzt wurden.

Zur Bestimmung des Ortes des hinteren Linsenscheitels bediente ich mich ausschliesslich des $\mathrm{Mi}$ kroskops. Bei Anwendung des letzteren benutzte ich, (wenn die Sonne nicht da war) auch die helle Flamme einer Gaslampe; das Licht der letzteren erweist sich stark genug für das Zustandekommen deutlicher und scharfer Reflexe von der hinteren Linsenfläche.

*) In die Tabelle wurden für die Bildgrösse (B) Mittelwerthe aus mehreren Beobachtungen eingeführt. 
Aus der Bildgrösse, der Objectgrösse und der Entfernung des Objectes vom Auge wurde $q$ berechnet die Brennweite des katroptrisch dioptrischen Systems von der hinteren Linsenfläche. Nach Berechnung von $q$ wurde der Radius der hinteren Linsenfläche nach der Formel $r=\frac{q\left(f_{2}-d\right)^{2}}{1 / 2 f_{1} f_{2}^{\prime}-q\left(f_{2}-d\right)} *$ berechnet, in der $f_{1}$ und $f_{2}$ die Brennweiten des Auges (aus dem schematischen Auge genommen) und d den Abstand der hinteren Linsenfläche vom zweiten Hauptpunkt des Auges (siehe die Einleitung) bedeuten.

Tabelle (III.) zur Bestimmung des Ortes und des Krümmungradius derhinteren Linsenfläthe.

H. H. H. G.

Ophth. Mikr. Ophth. Tikr. Ophth. Mikr.

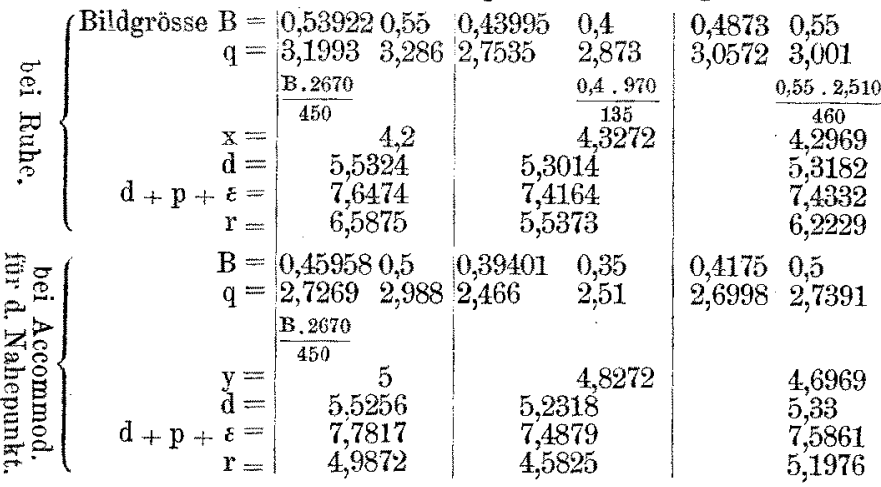

Bei den Berechnungen des Krümmungsradius der hinteren Linsenfläche und des Abstandes (d) der letzteren vom zweiten Hauptpunkte des Auges benutzte ich (nnit Ausnahme des Auges von H. S., für welches die mit dem Mikroskop gewonnenen Grössen benutzt wur-

*) Helmholtz. Arch. f. Ophtb. Bd. I. 2, pagr 19. 
den) für $q$ eine Grösse, die aus ophthalmometrischen Daten gewonnen wurde, da für letztere mehr Controlmessungen gemacht waren, und da die Sounenreflexe der hinteren Linsenfläche immer scharf und lichtstark, auch bei der Verdoppelung durch die Ophthalmometerplatten, erschienen. Ausserdem hatte eine absolut genaue Messung der Reflexbilder mit dem Mikrometer, bei einiger Beweglichkeit derselben und bei der noch nicht ganz genügenden Theilung des Mikrometers für mich doch einige Schwierigkeiten; so dass im gegebenen Fallo diejenigen Zahlengrössen für q, welche mit Hilfe des Ophthalmometers gewonnen sind, wohl noch genauer sind.

In der nächstfolgenden Tabelle sind die optischen Constanten und Cardinalpunkte der von mir untersuchten Augen zusammengestellt, mit Zugrundenahme bei der Berechnung der oben angeführten Zahlen des Helmhol tz'schen schematischenAuges. 
(Tabelle IV.) Optisehe Constanten und Car dinalpunktedreier Augen bei Ruhe und A c commodation.

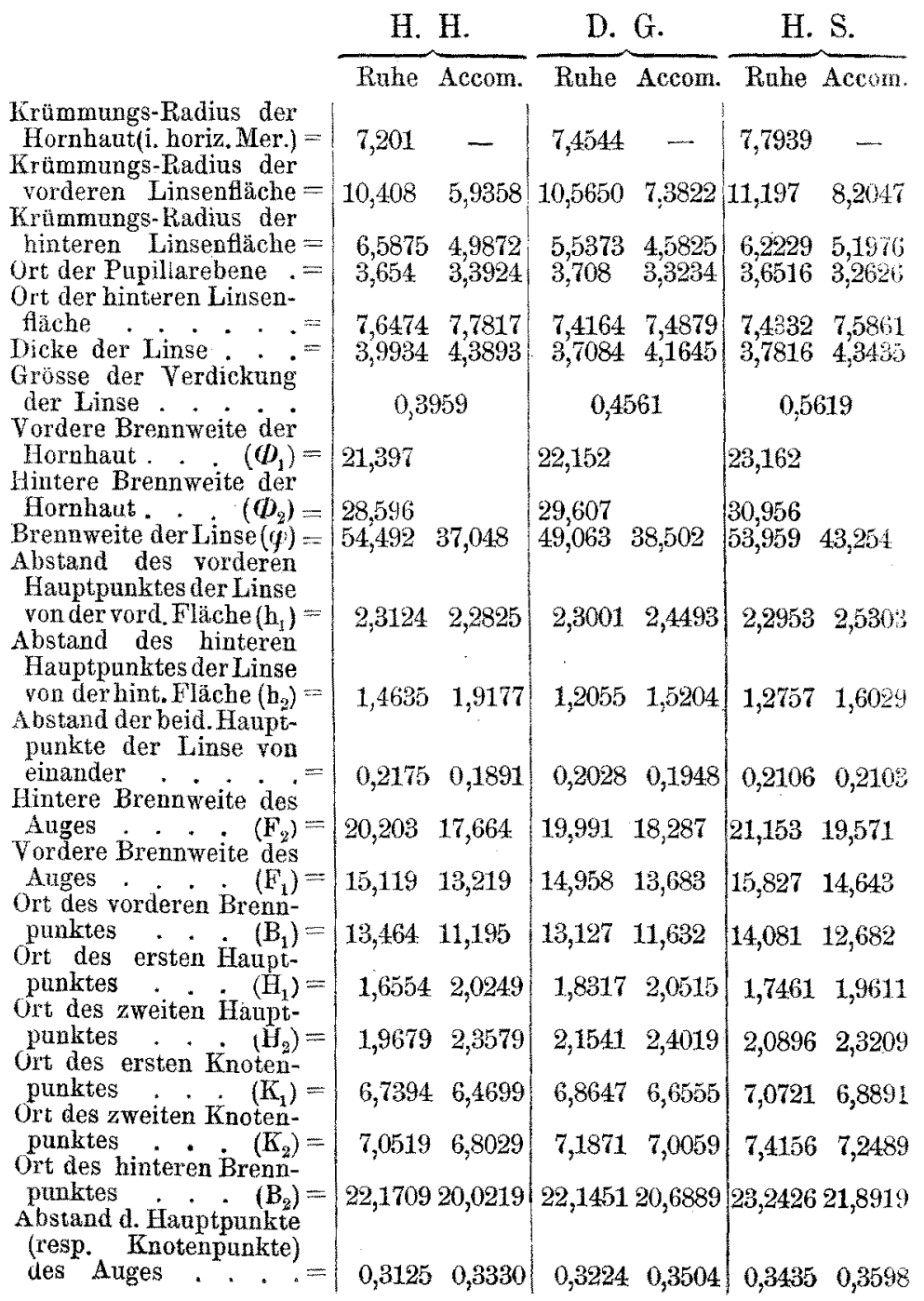




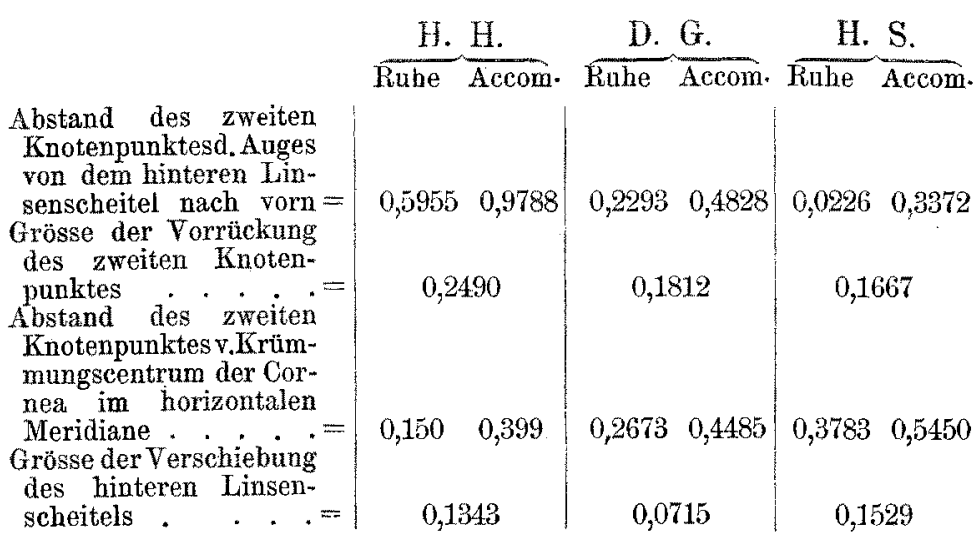

Aus dieser Tabelle ist ausser einigen Befunden (grösserer Brennweite und beträchtlicherer Dicke der Linse, grösserem Abstand des Ortes der hinteren Linsenfläche von der Hornhaut, weniger starkem Vorrücken des zweiten Knotenpunktes des Auges), welche wohl keiner ausführlicheren Besprechung bedürfen, der Umstand ersichtlich, dass auch in den von mir untersuchten Augen, so wie in den Augen von Schoeler und Mandelstamm, die hintere Linsenfläche bei der Accommodation ihren Ort verändert, und zwar nach binten zurückweicht (alle untersuchten Angen waren mehr oder weniger myopisch.) Die Grössen der Verschiebung $(0,1343$ - 0,0715 - 0,1529 Millim.) sind etwas kleiner, als in den Augen von Schoeler $(0,3188$ vorwärts) und M andelstamm $(0,3268$ rückwärts); in dem nur sehr schwach myopischen Auge $\left(M^{1 / 1}{ }^{0}\right)$ von $H$. G. fand ich nach der Berechnung die Grössen der Verschiebung $(0,0715$ Millim.) am geringsten, so dass man sagen kann, dass in diesem Auge der Ort der hinteren Linsenfläche sich fast gar nicht verändert hatte.

Weiter ersieht man, dass sich der Krümmungs- 
radius der hinteren Linsenfläche bei äusserst angestrengter Accommodation ziemlich bedeutend verkürzt; eine Verkürzung dieses Radius in solchem Grade kommt also, wie schon Schoeler und Mandelstamm mit Recht bemerken, wohl nicht ausschliesslich bei Presbyopen*) vor. Uebrigens finden wir eine verhältnissmässig bedeutende Zunahme der Krümmung der hinteren Linsenfläche auch schon in der von Woinow angegebenen Tabelle**), die sich nicht auf presbyopische Augen bezieht.

Ich habe Berechnungen angeführt, für welche sehr wichtige Daten (Abstände zwischen den brechenden Flächen) vermittelst eines neuen Instrumentes gewonnen sind. Diese Daten sind genauer, als die durch frühere Methoden bestimmten, und ersetzen theilweise solche Grössen, die man gewöhnlich aus dem schematischen Auge nahm; und dies ist auch ein Fortschritt der Ophthalmometrie. - Was nun überhaupt die Vorzüge der neuen (mikrooptometrischen) Untersuchungsmethode vor der ophthalmometrisehen betrifft, so sind dieselben von Schoeler und Mandelstamm ziemlich ausführlich besprochen. Meinerseits habe ich, wie aus den oben angeführten Bemerkungen schon zu ersehen ist, mich überzengt, dass zur genauen und verhältnissmässig raschen Bestimmung der Lage der Pupillarebene und des Ortes der hinteren Linsenfläche wir im Mikrooptometer ein ganz unersetzliches Instrument besitzen, welches wohl bald auch bei klinisehen Untersuchungen Verwerthung finden wird. Es bleiben nur immer eine absolut unbewegliche Aufstellung und einige technische Verbesserungen des Instrumentes zu wünschen.

Was aber die möglichst genaue Messung der von

*) Adamük und Woinow. Ueber die Accommodation der Presbyopen. Arch. f. Ophth. Bd. XVI. 1.

**) Klinische Monatsblatter f. Augenheilk. 1866, S. 474. 
den spiegelnden Flächen des Auges entworfenen Bilder betrifft, so muss bei der Schärfe und Helligkeit der Reflexe bei Anwendung von Sonnenlicht und hauptsächlich bei der Unabhängigkeit der Messung von den beständigen, wenn auch nur sehr geringen Schwankungen des Auges - vatürlich das Ophthalmometer vorgezogen werden.

Herrn Professor Helmholtz sage ich für die freundliche Belehrung und den vielfachen Rath, die mir von ihm während meiner Beschäftigung in seinem Laboratorium zu Theil wurden, meinen aufrichtigsten Dank. Berlin, Ende Februar 1874. 